\title{
Square of the degree of coherence and dispersion- induced pulse broadening in a fiber-optic transmission
}

\author{
Nori Shibata $^{1 \mathrm{a})}$, Kimitaka Watanabe ${ }^{1}$, Masaharu Ohashi ${ }^{2}$, \\ and Kazuhiko Aikawa ${ }^{3}$ \\ ${ }^{1}$ Nihon University, College of Engineering, Koriyama, Fukushima 963-8642, Japan \\ ${ }^{2}$ Osaka Prefecture University, Division of Electrical Engineering and Information \\ Science Graduate School of Engineering, Sakai, Osaka 599-8531, Japan \\ ${ }^{3}$ Advanced Technology Laboratory, Fujikura Ltd., Sakura, Chiba 285-8550, Japan \\ a)shibata@ee.ce.nihon-u.ac.jp
}

\begin{abstract}
We describe a method for measuring optical pulse broadening with continuous wave beam emitted from a low-coherence source. A twobeam interferometer composed of nondispersive and dispersive arms makes it possible to obtain the dispersion-induced pulse broadening. Experiments are carried out using a standard single-mode fiber and a two-mode fiber in the $1560 \mathrm{~nm}$-wavelength region. As a result, the square of the degree of coherence, $|\gamma|^{2}$, well reflects the dispersion-induced pulse broadening for an unchirped Gaussian pulse. The theoretically derived relationship between these two physical phenomena well reflects the experimentally obtained results. Chromatic dispersion values obtained by the method are in good agreement with those obtained by the alternative method. The salient feature of the present method is clearly shown.
\end{abstract}

Keywords: degree of coherence, pulse broadening, two-beam interferometer, differential group delay, chromatic dispersion

Classification: Optical Fiber for Communications

\section{References}

[1] N. Shibata, M. Tateda, S. Seikai, and N. Uchida, "Spatial technique for measuring modal delay differences in a dual mode optical fiber," Appl. Opt., vol. 19, pp. 1489-1492, 1980. DOI:10.1364/AO.19.001489

[2] M. Tateda, N. Shibata, and S. Seikai, "Interferometric method for chromatic dispersion measurement in a single-mode optical fiber," IEEE J. Quantum Electron., vol. 17, pp. 404-407, 1981. DOI:10.1109/JQE.1981.1071115

[3] H. T. Shang, "Chromatic dispersion measurement by white-light interferometry on meter-length single-mode optical fibers," Electron. Lett., vol. 17, pp. 603605, 1981. DOI:10.1049/el:19810424

[4] J. Stone and L. G. Cohen, "Minimum dispersion spectra of single-mode fibers measured with 0.1-ps resolution by white light cross-correlation," Electron. Lett., vol. 18, pp. 716-718, 1982. DOI:10.1049/el:19820487 
Douay, C. Lepers, A.-F. Obaton, and G. Melin, "Phase-sensitive optical lowcoherence reflectometry technique applied to the characterization of photonic crystal fiber properties," Opt. Lett., vol. 30, pp. 361-363, 2005. DOI:10.1364/ OL.30.000361

[6] P. Hamel, Y. Jaouën, R. Gabet, and S. Ramachandran, "Optical low-coherence reflectmetry for complete chromatic dispersion characterization of few-mode fibers," Opt. Lett., vol. 32, pp. 1029-1031, 2007. DOI:10.1364/OL.32.001029

[7] N. Shibata, M. Tsubokawa, T. Nakashima, and S. Seikai, "Temporal coherence properties of a dispersively propagating beam in a fiber-optic interferometer," J. Opt. Soc. Am. A, vol. 4, pp. 494-497, 1987. DOI:10.1364/JOSAA.4.000494

[8] K. Takada, N. Takato, J. Noda, and N. Uchida, "Interferometric optical-timedomain reflectometer to determine backscattering characterization of silicabased glass waveguides," J. Opt. Soc. Am. A, vol. 7, pp. 857-867, 1990. DOI:10.1364/JOSAA.7.000857

[9] S. Diddams and J.-C. Diels, "Dispersion measurements with white-light interferometry," J. Opt. Soc. Am. B, vol. 13, pp. 1120-1129, 1996. DOI:10.1364/ JOSAB.13.001120

[10] N. Shibata, A. Nakazono, and Y. Inoue, "Interference between two orthogonally polarized modes traversing a highly birefringent air-silica microstructure fiber," J. Lightw. Technol., vol. 23, no. 3, pp. 1244-1252, March 2005. DOI:10.1109/JLT.2005.843522

[11] W. A. Hamilton, A. G. Klein, and G. I. Opat, "Longitudinal coherence and interferometry in dispersive media," Phys. Rev. A, vol. 28, pp. 3149-3152, 1983. DOI:10.1103/PhysRevA.28.3149

[12] A. F. Fercher, C. K. Hitzenberger, M. Sticker, R. Zawadzki, B. Karamata, and T. Lasser, "Numerical dispersion compensation for partial coherence interferometry and optical coherence tomography," Opt. Express, vol. 9, pp. 610-615, 2001. DOI:10.1364/OE.9.000610

[13] K. Okamoto, Fundamentals of Optical Waveguides, 2nd ed. Elsevier/ Academic, 2006.

[14] N. Shibata, K. Watanabe, and M. Ohashi, "Chromatic dispersion diagnosis for the two-modes of few-mode photonic crystal fiber," IEEE Photon. Technol. Lett., vol. 28, no. 4, pp. 437-440, Feb. 2016. DOI:10.1109/LPT.2015.2498618

\section{Introduction}

Interferometric methods for measuring dispersive nature of fiber have been intensively studied since they make it possible to measure the differential group delay (DGD) between guided modes and/or the chromatic dispersion in short lengths of fiber $[1,2,3,4,5,6]$. Low-coherence light sources are usually used to measure the group delay dispersion with sub-picosecond resolution. The temporal coherence degrades when the interference involves two waves with different group velocity dispersions (GVDs) $[7,8,9,10]$. The influence is on the measurement accuracies of DGD and chromatic dispersion, since the curve shape of the degree of coherence, $|\gamma|$, with respect to the interference broadens as the GVD and fiber length increase. Furthermore, it has been pointed out that the detection sensitivity of a fiber-optic sensor is influenced by the temporal coherence response [10]. Therefore, the loss of temporal coherence cannot be ignored if we use two-beam interferometers that have optical paths with different dispersion characteristics. On the other hand, there is the potential to change the detrimental feature to a beneficial one if we can find an 
analogy between the degree of coherence and the dispersion-induced effect such as an optical pulse broadening. As was introduced in Ref. [7], Hamilton et al. [11] analyzed the coherence properties of a dispersively propagating wave such as electrons or neutrons in vacuo, or light in a medium, and discussed the observation of its spatial and temporal coherence properties by means of a two-beam interferometer. A numerical dispersion compensation technique has been also presented for optical coherence tomography [12]. These theoretical treatments have been useful, even though no experimental result has been displayed.

In this article, we theoretically and experimentally investigate the relationship between the degradation of temporal coherence caused by fiber chromatic dispersion and dispersion-induced pulse broadening. We perform experiments using a standard single-mode fiber (SMF) and a two-mode fiber (TMF) which are inserted as the dispersive path in a two-beam interferometer. The square of the degree of coherence $|\gamma|^{2}$ is found to reflect the dispersion-induced pulse broadening. We compare the GVD and chromatic dispersion values that we obtained experimentally with those we obtained using an alternative method.

\section{Experimental arrangement and theoretical background}

We consider a two-beam interferometer with one arm composed of a nondispersive medium and the other of a dispersive medium. Fig. 1 shows the interferometer we used to measure the dispersion-induced pulse broadening by evaluating the degree of coherence. The light from a low-coherence source (LCS) is coupled to a singlemode fiber $\mathrm{SMF}_{1}$ to obtain a point light source. The beam emitted from $\mathrm{SMF}_{1}$ is collimated and divided into two beams with a half-mirror $\mathrm{HM}_{1}$. The light beam is intensity modulated by a mechanical chopper $\mathrm{MC}$ inserted between $\mathrm{SMF}_{1}$ and $\mathrm{HM}_{1}$. The modulated beam illuminates a two-beam interferometer composed of a test fiber and an air path. The direction of the beam in the air is changed by mirrors $M_{1}$ and $M_{2}$, and is superposed on the beam emitted from the test fiber by a half mirror $\mathrm{HM}_{2}$. Another polarizer $\mathrm{P}_{2}$ is adjusted to give maximum interference visibility. The interference signal appears as a chopped signal at a pin-photodiode (pin-PD), and this signal component is detected with a lock-in amplifier. The amplifier output gives the intensity distribution yielded by interference for the guided waves and a polarized wave propagating in the air. The intensity distributions are measured by moving mirror $\mathrm{M}_{3}$ backward. If the two beams have the same intensities, the intensity $I$ at the pin-PD is expressed as [10],

$$
I=I_{0}[1+\operatorname{Re}(\gamma)],
$$

where $\gamma$ represents the complex degree of coherence. The visibility $V$ of interference fringes defined as $V=\left(I_{\max }-I_{\min }\right) /\left(I_{\max }+I_{\min }\right)$, which equals the degree of coherence $|\gamma|$, is measured as a function of the nondispersive path difference $2 d$. Here $I_{\max }$ and $I_{\min }$ denote the maximum and minimum intensities of the interference fringes, respectively.

In accordance with the previous theoretical treatments [7, 10], we analyzed the coherence properties of a dispersively propagating beam transmitted through the fiber with length $L$ and chromatic dispersion $D$ shown in Fig. 1. We derive the degree of coherence $|\gamma|$ as a function of $2 d$ for a single Gaussian spectral function 


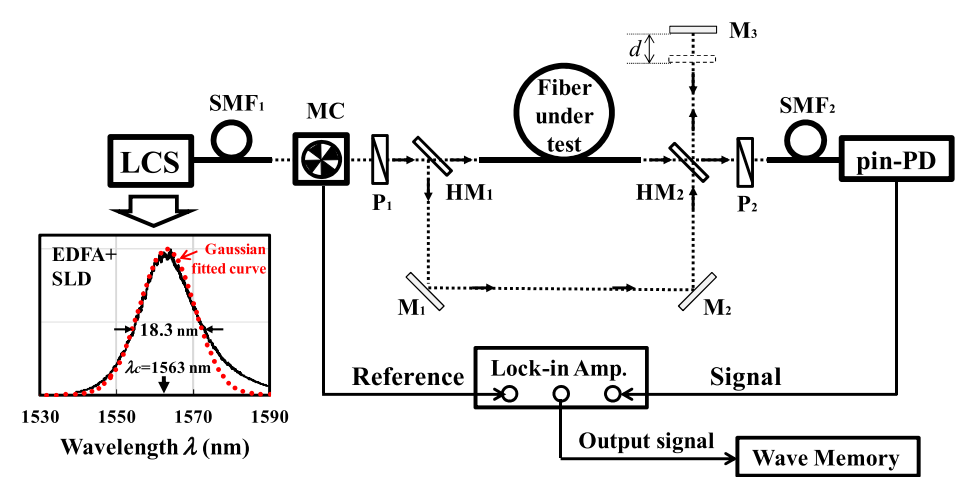

Fig. 1. Experimental arrangement for measuring the dispersioninduced pulse broadening by evaluating the degree of coherence.

given as $S(\omega)=\exp \left[-\left(\omega-\omega_{c}\right)^{2} / 2 \Delta \omega^{2}\right] /(2 \pi)^{1 / 2} \Delta \omega$, where $\omega$ is the angular frequency, $\omega_{\mathrm{c}}$ the center angular frequency, and $\Delta \omega$ the spectral half-width. The complex degree of coherence $\gamma(\tau)$ is derived as follows [10]

$$
\gamma(\tau)=\frac{\exp \left[i \omega_{\mathrm{C}}\left\{\left(1 / v_{\mathrm{P}}-1 / c\right) L-2 d / c\right\}\right]}{\sqrt{1-i \Delta \omega^{2} \beta_{2} L}} \exp \left[-\frac{(\Delta \omega \tau)^{2}}{2\left(1-i \Delta \omega^{2} \beta_{2} L\right)}\right]
$$

and

$$
\tau=\left(1 / v_{\mathrm{g}}-1 / c\right) L-2 d / c
$$

where $v_{\mathrm{p}}$ and $v_{\mathrm{g}}$ are the respective phase and group velocities of a guided mode, $c\left(=3 \times 10^{8} \mathrm{~m} / \mathrm{s}\right)$ is the velocity of light in free space, and $\beta_{2}\left(=\mathrm{d}^{2} \beta / \mathrm{d} \omega^{2}\right)$ is the GVD parameter where $\beta$ is the propagation constant. The degree of coherence $|\gamma(\tau)|$ is then derived from Eq. (2) as follows,

$$
|\gamma(\tau)|=\frac{1}{\sqrt[4]{1+\left(\beta_{2} L / \tau_{\mathrm{coh}^{2}}\right)^{2}}} \exp \left[-\frac{\tau^{2}}{2 \tau_{\operatorname{coh}^{2}}^{2}\left\{1+\left(\beta_{2} L / \tau_{\mathrm{coh}^{2}}\right)^{2}\right\}}\right]
$$

where $\tau_{\text {coh }}=1 / \Delta \omega$ is the coherence time. When $\beta_{2}=0$, Eq. (4) is simplified to give

$$
|\gamma(\tau)|=\exp \left[-\tau^{2} /\left(2 \tau_{\mathrm{coh}^{2}}{ }^{2}\right] .\right.
$$

Next, we describe the dispersion-induced pulse broadening of unchirped Gaussian pulses. Optical pulses with a Gaussian envelope of the form $|E(t)|^{2}=$ $\exp \left\{-\left(t / T_{\text {in }}\right)^{2}\right\}$ are launched into a fiber. Here $E(t)$ is the electromagnetic field, $t$ is the time and $T_{\text {in }}$ is the incident pulse width. For a Gaussian pulse, $T_{\text {in }}$ is related to the full width at half maximum (FWHM) pulse width $T_{\mathrm{FWHM}}$ by $T_{\mathrm{FWHM}}=$ $(2 \ln 2)^{1 / 2} T_{\text {in }}$. The intensity $|E(z, t)|^{2}$ at the exit face of the fiber with length $L$ is given by [13]

$$
|E(L, t)|^{2}=\frac{1}{\sqrt{1+\left(\beta_{2} L / T_{\text {in }}^{2}\right)^{2}}} \exp \left[-\frac{\left(t-L / v_{g}\right)^{2}}{T_{\text {in }^{2}}{ }^{2}\left\{1+\left(\beta_{2} L / T_{\text {in }^{2}}\right)^{2}\right\}}\right]
$$

The output pulse width $T_{\text {out }}$ is given as

$$
T_{\text {out }}=T_{\text {in }}\left[1+\left(\beta_{2} L / T_{\text {in }}^{2}\right)^{2}\right]^{1 / 2}
$$




$$
|\gamma(\tau)|^{2}=\frac{1}{\sqrt{1+\left(\beta_{2} L / \tau_{\mathrm{coh}^{2}}\right)^{2}}} \exp \left[-\frac{\tau^{2}}{\tau_{\operatorname{coh}^{2}}^{2}\left\{1+\left(\beta_{2} L / \tau_{\mathrm{coh}^{2}}\right)^{2}\right\}}\right] .
$$

The forms of Eqs. (6) and (8) suggest the equivalence of these two equations, when

$$
T_{\text {out }} / T_{\text {in }}=1+\left(\beta_{2} L / \tau_{\mathrm{coh}^{2}}\right)^{2} .
$$

From Eq. (9), we have

$$
\beta_{2}=\left(\tau_{\mathrm{coh}}^{2} / L\right)\left(T_{\text {out }} / T_{\text {in }}-1\right)^{1 / 2} .
$$

Replacing $T_{\text {out }}$ and $T_{\text {in }}$, respectively, with the FWHM widths $\tau_{\text {out }}$ and $\tau_{\text {in }}$ observed in the $|\gamma|^{2}$ response, we obtain

$$
\beta_{2}=\left(\tau_{\mathrm{coh}}{ }^{2} / L\right)\left(\tau_{\text {out }} / \tau_{\text {in }}-1\right)^{1 / 2} .
$$

Since the chromatic dispersion $D$ is related to $\beta_{2}$ as $D=-\left(2 \pi c / \lambda^{2}\right) \beta_{2}$ where $\lambda$ is the wavelength, we can estimate $D$ using the measured values of $\tau_{\text {out }}$ and $\tau_{\text {in }}$. It should be noted here that $\tau_{\text {in }}$ corresponding to the incident pulse width is measured for the interference of waves traversing the two nondispersive arms in a balanced Michelson interferometer [9]. Eq. (5) is valid for the interference, and $\tau_{\text {coh }}$ is related to $\tau_{\text {in }}$ as $\tau_{\text {coh }}=\tau_{\text {in }} / 2(\ln 2)^{1 / 2}$.

\section{Experimental results}

We used an LCS that employed an $\mathrm{Er}^{3+}$-doped fiber amplifier (EDFA) and a superluminescent diode (SLD) operating at $1550 \mathrm{~nm}$. The emitted light spectrum of the EDFA-assisted SLD and its Gaussian fitting curve are shown in the inset of Fig. 1. The emitted spectrum matches the Gaussian fitted curve well, and the FWHM spectral width $\Delta \lambda_{\text {FWHM }}$ is $18.3 \mathrm{~nm}$ at a center wavelength $\lambda_{\mathrm{c}}$ of $1563 \mathrm{~nm}$. We prepared two test fibers. One was an SMF with an effective cutoff wavelength of $1247 \mathrm{~nm}$ for the $\mathrm{LP}_{11}$ mode. The other was a TMF whose effective cutoff wavelengths were $1727 \mathrm{~nm}$ and $1084 \mathrm{~nm}$ for the $\mathrm{LP}_{11}$ and $\mathrm{LP}_{21}$ modes, respectively.

Fig. 2 shows the intensity distributions and $|\gamma|^{2}$ as a function of time $\tau$. In Fig. 2, the intensity $I_{0}$ corresponds to that expressed in Eq. (1). Fig. 2(A) shows the intensity distribution we obtained for the interference of waves traversing the two air paths in a Michelson interferometer, and $|\gamma(\tau)|^{2}$ as a function of $\tau$. The experimentally obtained and theoretically predicted results were in good agreement with for the magnitudes of $|\gamma(\tau)|^{2}$, and the shape of the relevant curve as a function of $\tau$. Fig. 2(A) gives the FWHM width $\tau_{\text {in }}=0.273 \mathrm{ps}$ corresponding to the fiber launched pulse-width. Then the coherence time of $\tau_{\mathrm{coh}}=0.164 \mathrm{ps}$ was obtained experimentally. Figs. 2(B)-(E) show the time dependences of the intensity distributions and $|\gamma(\tau)|^{2}$ for $1.0 \mathrm{~m}$ - and $1.3 \mathrm{~m}$-long test fibers. With the SMF, the $\tau_{\text {out }}{ }^{-}$ value increases with $L$ and the modulus of $|\gamma(\tau)|^{2}$ at $\tau=0$ decreases with $L$. Using Eqs. (11), we can obtain average values of $\left|\beta_{2}\right|=(2.09 \pm 0.03) \times 10^{-2} \mathrm{ps}^{2} / \mathrm{m}$. Then we obtained $D=16.1 \pm 0.3 \mathrm{ps} / \mathrm{km} / \mathrm{nm}$. On the other hand, Figs. 2(D) and (E) show that the $\mathrm{LP}_{01}$ modes are 0.922 and $1.160 \mathrm{ps}$ behind the $\mathrm{LP}_{11}$ modes at $L=1.0$ and $1.3 \mathrm{~m}$, respectively. We obtained an average DGD value of $-907.2 \pm 14.8 \mathrm{ps} / \mathrm{km}$. The chromatic dispersion characteristics of the $\mathrm{LP}_{01}$ and $\mathrm{LP}_{11}$ modes are obtained from the $\tau_{\text {out }}-\mathrm{value}$ as a function of $L$, and the experimentally estimated $\left|\beta_{2}\right|$ values are $(2.24 \pm 0.09) \times 10^{-2}$ and $(7.46 \pm 0.75) \times 10^{-3}$ 
$\mathrm{ps}^{2} / \mathrm{m}$, respectively. Then we obtained the respective chromatic dispersions of $D_{01}=17.3 \pm 0.5$ and $D_{11}=5.8 \pm 0.5 \mathrm{ps} / \mathrm{km} / \mathrm{nm}$. It is interesting that $D_{11}$ is much smaller than $D_{01}$ at $\lambda_{\mathrm{c}}=1563 \mathrm{~nm}$.
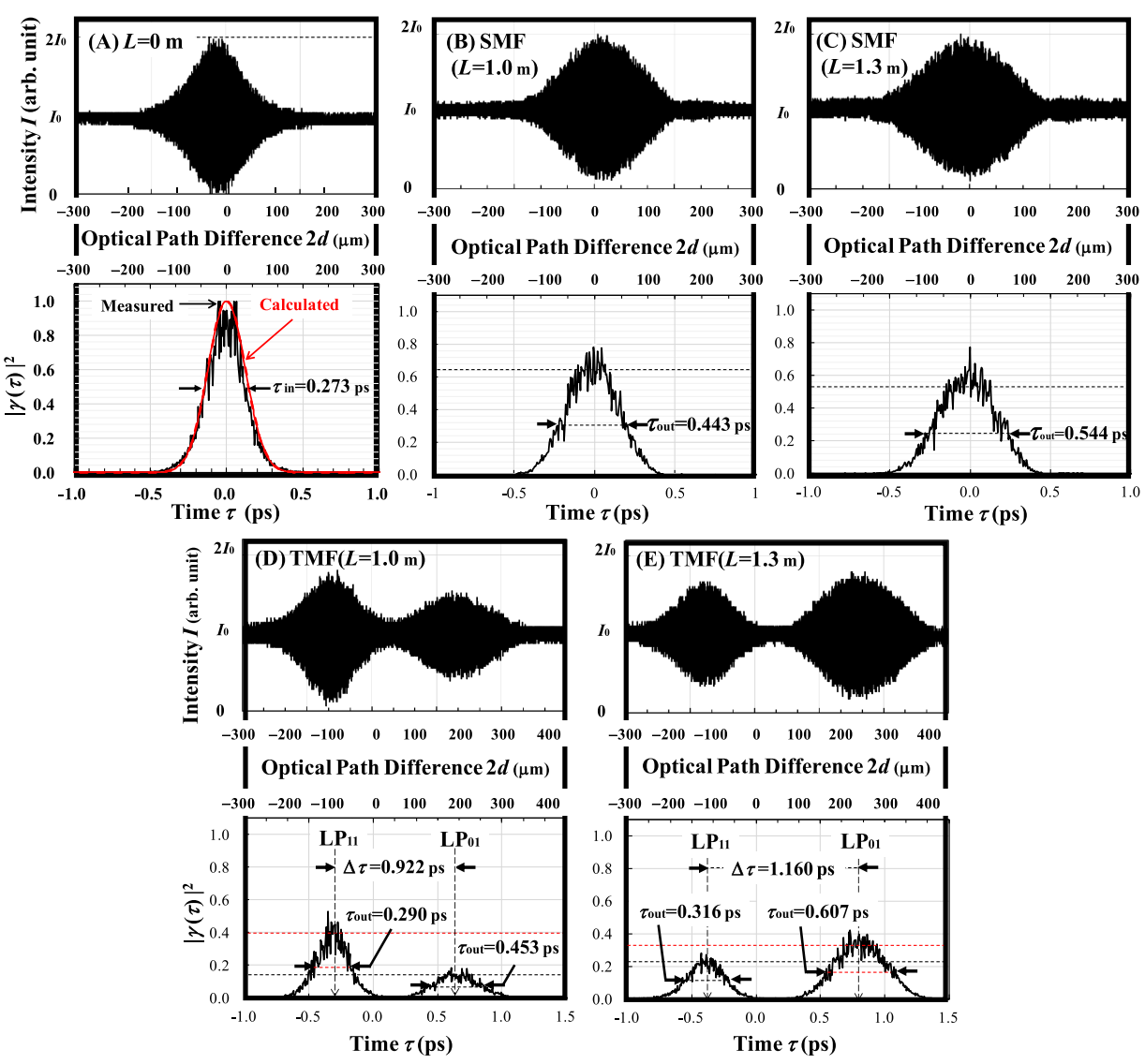

Fig. 2. Intensity distributions and $|\gamma(\tau)|^{2}$ as a function of time $\tau$ for (A) a balanced Michelson interferometer, (B) $1.0 \mathrm{~m}$-long SMF, (C) $1.3 \mathrm{~m}$-long SMF, (D) $1.0 \mathrm{~m}$-long TMF, and (E) $1.3 \mathrm{~m}$-long TMF.
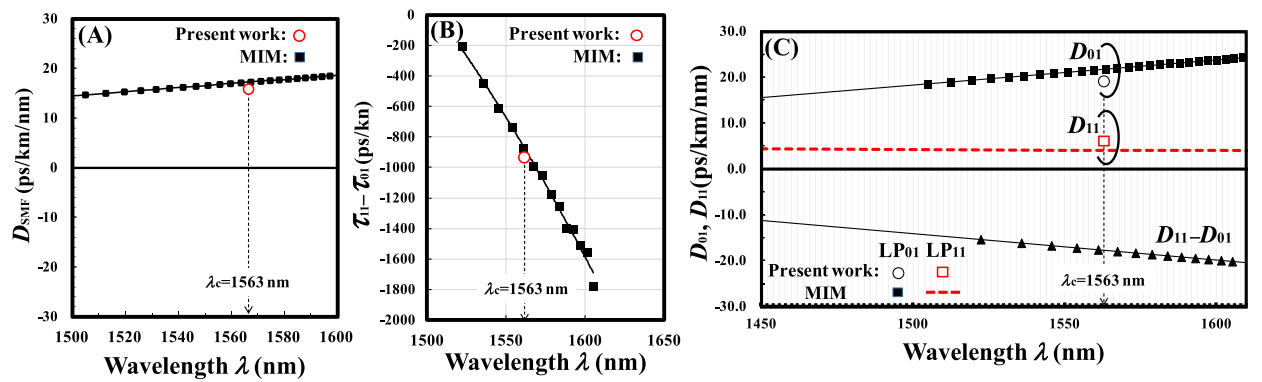

Fig. 3. Comparison of the values estimated with the present method and MIM for (A) chromatic dispersion of the SMF, (B) DGD of the TMF, and (C) $D_{01}$ and $D_{11}$ for the TMF.

A modal interferometer method (MIM) [14] was used to confirm the validity of the present method. Figs. 3(A), (B), and (C) show the wavelength dependences of the chromatic dispersion of the SMF, and the DGD and chromatic dispersions ( $D_{01}$ and $\left.D_{11}\right)$ of the TMF, respectively. Open and filled symbols represent the results 
obtained with the present method and MIM, respectively. Fig. 3 shows that the experimental results obtained with the present method well reflect those obtained by the MIM at the $1563 \mathrm{~nm}$. It is noteworthy that the $\mathrm{LP}_{11}$ mode of the TMF has dispersion-flattened characteristics for the dispersion-slope of $\mathrm{d} D_{11} / \mathrm{d} \lambda=-2.8 \times$ $10^{-3} \mathrm{ps} / \mathrm{km} / \mathrm{nm}^{2}$ in the telecommunication band.

\section{Conclusions}

We investigated the analogy between the loss of the degree of coherence and dispersion-induced pulse broadening. The theoretically derived relationship between these two physical phenomena well reflects the experimentally obtained results. Furthermore, the validity of the present method is confirmed by employing the modal interferometer method as an alternative approach. The obtained experimental results are in good agreement. The salient feature of the present method is that it makes it possible to estimate both the chromatic dispersion of each mode and the DGD among the guided modes traversing few-mode fibers. 